\title{
Material selection combined with optimal structural design for mechanical parts*
}

\author{
Le-miao QIU, Liang-feng SUN ${ }^{\dagger}$, Xiao-jian LIU, Shu-you ZHANG \\ (State Key Laboratory of Fluid Power Transmission and Control, Zhejiang University, Hangzhou 310027, China) \\ ${ }^{\dagger}$ E-mail: tjslf@163.com \\ Received Jan. 1, 2013; Revision accepted Apr. 7, 2013; Crosschecked May 16, 2013
}

\begin{abstract}
To reduce the environmental impact of mechanical parts, an approach integrating structural design and material selection was studied. Adding the discrete variable of material, a hybrid optimization model was built with the aim of minimizing environmental impact and based on an ordinary structure optimization model. An optional material set was built by combining measures of qualitative and quantitative screening, while the lifecycle environmental impact of the materials was quantified using the method of Eco-indicator 99. Two groups of structurally optimal solutions were calculated with ideal and negative-ideal materials selected respectively, and then the hybrid model was simplified by comparing the solutions. A material environmental performance index was calculated using an analytic method. By comparing this index for every material in the optional material set, the optimal material can be found and the structural solutions calculated. This method was applied to a dowel bar design process as a case study. The results show that the environmental impact of each material has a significant effect on the optimal structural solution, and it is necessary to study the integration of structural design and material selection.
\end{abstract}

Key words: Green design, Structure optimization, Material selection, Environmental performance index doi:10.1631/jzus.A1300004

Document code: A

CLC number: TH122

\section{Introduction}

With more attention being paid to the environment, the awareness of green design has been increasingly reflected in the detailed design process. Material selection and structural design are two important aspects of the detailed design stage. The selection of eco-friendly materials and the optimization of the structure are very effective methods to reduce the environmental impact of mechanical parts (Almeida et al., 2010; Kurk and Eagan, 2008; Chu et al., 2009). Generally, the processes of material selection and optimal structural design are carried out separately. The majority of material selection studies

\footnotetext{
${ }^{\ddagger}$ Corresponding author

* Project supported by the National Natural Science Foundation of China (No. 51275458), and the Zhejiang Provincial Natural Science Foundation of China (No. LY12E05019)

(c) Zhejiang University and Springer-Verlag Berlin Heidelberg 2013
}

focus upon selecting the most appropriate material under various performance constraints for a given engineering design. Deng and Edwards (2007) analyzed the importance of material identification and selection for engineering design problem solving, and provided a summary of the latest research efforts in this area. Ashby (2000) proposed a multi-objective optimization method for material selection with several conflicting objectives. Rao (2007), Rao and Davim (2008), and Rao and Patel (2010) analyzed a multiple attribute decision-making method in material selection and built a decision-making framework model based on a combined TOPSIS (technique for order preferenceby similarity to ideal solution) and AHP (analytic hierarchy process) method. Considering the fuzzy characteristics of material properties, Khabbaz et al. (2009) introduced a fuzzy logic approach to deal with the qualitative properties of materials, and simplified the selection process. Hambali et al. (2010) introduced an analytical hierarchy 
process for material selection for a polymer-based composite bumper beam. Jahan et al. $(2010 ; 2011)$ proposed two methods of material selection with VIKOR (VIseKriterijumska Optimizacija I Kompromisno Resenje in Serbian) and material selection based on ordinal data. There are also many publications that concentrate on material selection taking into account environmental concerns (Holloway, 1998). Weaver et al. (1996) proposed a method to reduce environmental impact by material selection. Sun et al. $(2003 ; 2005)$ simplified product life cycle assessment by classifying materials according to their environmental and material properties. Ribeiro et al. (2008) presented a life cycle engineering methodology applied to material selection. Some knowledge-based systems have also been developed for material selection. Sapuan and Abdalla (1998) and Sapuan (2001) developed a prototype system for material selection of polymer-based composites for automotive components using a knowledge-based system tool-kit. Amen and Vomacka (2001) developed a case-based reasoning tool for material selection. Zha (2005) studied the process and material selection in concurrent product design for a manufacturing environment and developed a web-based advisory system. These methods can help designers to choose the material which can best meet specified design requirements. However, without taking into account the structure of the mechanical parts the structural design may not be optimized:

1. The material might be overkill. Its specific structure and properties may not be fully utilized, resulting in wastage.

2. Some properties of the material might not meet the specific structural requirements and the dimension may have to be enlarged, resulting in a heavy structure.

With the aim of reducing the mass of the mechanical parts, most structural optimization studies focus on searching for dimension optimization solutions using mathematical programming or digital approximation methods with a determined material (Peter and Anders, 2009). Song and Baldwin (1999) proposed a node-based structural shape optimization method which allowed large shape changes to achieve the optimal shape for planar structures. Wall et al. (2008) constructed a structural shape optimization framework based on an isogeometric analysis ap- proach. Nagy et al. (2010) presented a method of structural sizing and shape optimization of curved beam structures using an isogeometric approach. However, the solution spaces have been limited for a given material because different materials can affect the performance of mechanical parts. Therefore, it is necessary to study the integration of structural design and material selection. Several studies have been published concerning an integrated design approach. Ashby and Johnson (2002) developed a performance index which reflects the effect of a material on a structure for a given situation. Based on the performance index, Huang et al. (2009) developed a life cycle environmental performance index to investigate the relationship between the environmental impact of the material and structural constraints. By ranking candidate materials with the index, the optimal material can be found. But this method is effective only for simple design problems with a single variable. Natalia et al. $(2002 ; 2004)$ constructed a system to perform structural optimization of a chosen component based on the multipoint approximation method, with response surface fitting combined with MSC.MARC finite element analysis (FEA) code. But this method is very complex when used to analyze the optimal structure for each material using the finite element method.

In this paper, we integrate the processes of material selection and optimal structural design. With the aim of minimizing the environmental impact, a hybrid optimization model is constructed. An approach for obtaining the optimal structural dimensions and materials simultaneously is demonstrated. Unlike previous studies which aimed to reduce environmental impact by material selection or optimal structural design separately, this new approach can provide a more effective method by integrating these two processes. The structure and material of parts can be matched more effectively to avoid wastage.

\section{Hybrid discrete optimization model com- bining structural optimization and material selection}

By adding the discrete variable of material, a hybrid optimization model can be built based on a traditional structure optimization model. The optimal 
dimension and material of a structure can be obtained simultaneously through this model, with the aim of minimizing the environmental impact. The details of the process for building this model are as follows:

1. Establishing the objective function

Generally, the aim of a traditional structure optimization model for mechanical parts is to calculate the optimal structure dimension for the lightest weight. But the lightest weight for a mechanical part does not always guarantee minimum environmental impact if the ecological characteristics of the material are considered. Accordingly, the objective function should be:

$$
\min E=E_{\mathrm{IP}} \rho V,
$$

where $E$ is the elastic modulus, $E_{\mathrm{IP}}$ is the Eco-indicator of the material determined by the type of material, $\rho$ is the density of the material also determined by the type of material, and $V$ is the volume of the parts determined by the dimension of the structure.

\section{Determining the variables}

Some of the structural dimensions are fixed to meet functional requirements or assembly constraints, while others which can be changed are taken as the design objects. These structural dimensions are continuous variables denoted by $x_{i}$ and their value ranges are $\left[x_{i \min }, x_{i \max }\right]$, determined by various design constraints. The type of material is taken as a discrete variable $M$ and its value range is the optional material set $\left\{m_{1}, m_{2}, \ldots, m_{s}\right\}$ which consists of all the feasible materials screened from the material library by different performance requirements. Details of the process of screening the materials will be given in Section 3. Various properties of materials are discrete components of the material variable $M$ which influences the objective function directly. Generalized attribute function clusters are denoted by $f_{i}(M)$ to express these discrete components of the material. $f_{i}(M)$ reflects the mapping relation between material variable $M$ and the properties. For example, the elastic modulus $E$ is expressed as $f_{E}(M)$. Therefore, the complete variable space can be obtained combining $n$ continuous variables and one discrete variable consisting of various discrete components:

$$
\boldsymbol{X}:\left\{x_{1}, x_{2}, \ldots, x_{n},\left(f_{\rho}(M), f_{E_{\mathrm{IP}}}(M), f_{E}(M), \ldots\right)\right\} .
$$

\section{Determining the constraints}

Constraints derived from various functional and performance requirements of the mechanical part can be divided into two categories: qualitative constraints and quantitative constraints. Qualitative constraints are generally converted into requirements for the type of material and are used for preliminary screening of the materials set, while quantitative constraints, through which the structural performance mathematical inequality model can be established, are used for further screening. In a specific parts structural design problem, the quantitative constraints are divided into constraints of structural performance like stiffness and strength, and constraints of the dimension of the structure, which are the value ranges of the structural dimensions $x_{i}$.

Then, the hybrid optimization model for mechanical parts in a green design can be built as follows:

$$
\left\{\begin{array}{l}
\min f_{E_{\mathrm{IP}}}(M) \cdot f_{\rho}(M) \cdot V\left(x_{1}, x_{2}, \ldots x_{n}\right), \\
\text { s.t. } g_{j}(\boldsymbol{X}) \leq 0 \& \& M \in U_{k}, \\
\boldsymbol{X}=\left[x_{1}, x_{2}, \ldots x_{n}, M\right]^{\mathrm{T}} \\
x_{i \min } \leq x_{i} \leq x_{i \max } \\
M \in\left\{m_{1}, m_{2}, \ldots m_{s}\right\}
\end{array}\right.
$$

where $g_{j}(\boldsymbol{X}) \leq 0$ are the quantitative constraints of the structural performance, $M \in U_{k}$ are the qualitative constraints of the material type, $x_{i \min } \leq x_{i} \leq x_{i \max }$ are the constraints of the dimension of the structure, and $\left\{m_{1}, m_{2}, \ldots, m_{s}\right\}$ is the optimal material set.

This model combines continuous dimension variables and discrete material components. Therefore, searching for the optimal solution is a constrained nonlinear discrete optimization problem. The individual optimal solutions of the discrete components of the material cannot be the final solution directly because they may not the components of the same material.

\section{Process for construction of the optional material set}

\subsection{Preliminary screening of the material set}

To choose materials for mechanical parts, designers generally first filter the type of material according to the functional requirements, environmental requirements, shape forming technology, and so on. Then, materials which cannot satisfy the quantitative 
constraints, such as the allowable stress or allowable strain, will be removed to construct an optional material set (Fig. 1).

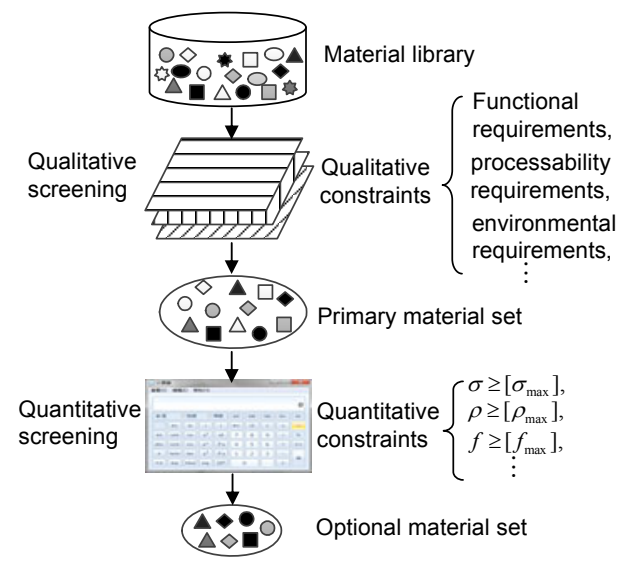

Fig. 1 Construction process for the optional material set

\section{Qualitative screening}

With advances in materials science, the range of materials and classification methods has increased. According to their chemical composition, the materials can be classified into metallic, inorganic nonmetallic, polymeric, or synthetic materials. According to their functional performance, they can be classified into structural or functional materials. According to their physical properties, the materials can be classified into conductive, insulating, magnetic, or high temperature materials. Therefore, the material library can be divided into various collections using different classification methods like $\left\{A_{1} \mid\right.$ Metal $\},\left\{A_{2} \mid\right.$ Polymer $\}$, $\left\{A_{3} \mid\right.$ Synthetic $\},\left\{B_{1} \mid\right.$ Conductive $\},\left\{B_{2} \mid\right.$ Magnetic $\}$, and so on.

These classification methods can effectively reduce the search range for material selection after confirming the classification of the material needed. The qualitative screening conditions can be expressed as

$$
M \in A_{2} \& \& M \in B_{2} \& \& \cdots
$$

\section{Quantitative screening}

The quantitative constraints of the structural performance $g_{j}(X) \leq 0$ can be transformed into the form $f_{i}(M) \geq Y_{i}\left(x_{1}, x_{2}, \ldots, x_{n}\right)$ in which the material performance components $f_{i}(M)$ are separated. $Y_{i}\left(x_{1}, x_{2}, \ldots, x_{n}\right)$ is a function with structure dimensions $x_{i}$ as its variables. The extreme value for this function and the quantitative screening conditions can be calculated from:

$$
f_{E}(M) \geq \delta_{1} \& \& f_{G}(M) \geq \delta_{2} \& \& \cdots .
$$

Reducing the search range of the suitable material with qualitative methods is more efficient but using quantitative methods is more accurate. These two kinds of methods follow the conventional ways to select the material. With the screening conditions Eqs. (4) and (5), the optional material set can be searched from the material library using the feasible set search algorithm. The optional material set $\left\{m_{1}\right.$, $\left.m_{2}, \ldots, m_{s}\right\}$ is prepared for further optimization design to facilitate selection.

\subsection{Quantitative environmental impact of materials}

Different materials cause different environmental impacts which affect the choice of materials for mechanical parts (Jahan et al., 2011). Therefore, it is necessary to quantify the environmental impact of each material. The assessment of the impact on the environment should take account of the whole life cycle of the material, including the processes of production, manufacturing, usage, recycling, and disposal of the material. Eco-indicator 99 (Rydh and Sun, 2005) is a method widely used for life cycle impact assessment. It can help designers to make an environmental assessment of the material from three aspects: damage to resources, damage to human health, and damage to ecosystem quality (Fig. 2). The main steps are:

1. Construct the process model. Identify the main processes of the material, from production of the raw material to disposal of the material, which cause direct or indirect effects on the environment.

2. Bill analysis. Collect all kinds of data related to the environment in the process and classify them into different categories according to three aspects: damage to resources, damage to human health, and damage to ecosystem quality.

3. Influence and damage analysis. For each specific environmental issue, calculate the Eco-indicator value using the collected data with reference to various standards. As shown in Fig. 2, Ri, Hi, and $E i$ are the Eco-indicator values of resources, human health, and ecosystem, respectively. $D_{R i}, D_{H i}$, and $D_{E i}$ are the 
data of different issues of resources, human health, and ecosystem, respectively. $D_{R}, D_{H}$, and $D_{E}$ are the standard reference data of different issues of resources, human health, and ecosystem, respectively.

4. Standardization and weighting. Normalize all types of Eco-indicator values and weight the three damage categories so that the Eco-indicator of the material can be obtained from their weighted sum. $W_{R}$ $W_{H}$, and $W_{E}$ are the weights of resources, human health, and ecosystem, respectively (Fig. 2).

The Eco-indicator of the material is the quantization value of the environmental impact caused by the material. It is represented as $E_{\mathrm{IP}}$ with the unit $\mathrm{Pt} / \mathrm{kg}$.

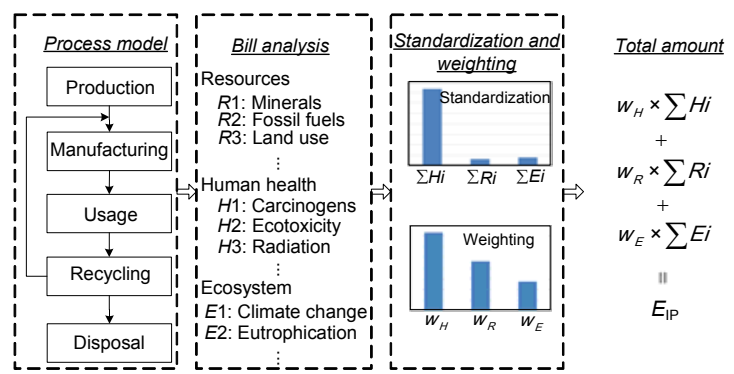

Fig. 2 Quantitative environmental impact of material using Eco-indicator 99

\section{Simplify the model and search for the op- timal solution}

The structure dimension continuous variables are coupled with the discrete material components, not the material $M$ as a whole variable. Therefore, it is necessary to put all the material components together to form the combination expression for $M$, namely the environmental performance index defined by Huang et al. (2009). The dimension variables and the material variable can then be divided and the optimal solution can be calculated. If the model is easy enough, the dimension optimal solutions in analytical form can be obtained by analytic transformation setting the material as constant. The environmental performance index can then be obtained by substituting the dimension optimal solutions into the objective function. If calculating the dimension optimal solutions in analytical form is difficult, it may be necessary to simplify the model after analyzing the objective function and the constraints. The details of the process are as follows.
Step 1: Take the best material properties and the worst material attribute for the mechanical part to make up the ideal material $M_{\mathrm{BEST}}$ and the negative ideal material $M_{\text {WORST }}$, respectively (Fig. 3). The ideal and negative ideal materials are virtual materials which do not belong to the optional material set. They are the theoretical boundaries of the material variable.

Step 2: Assume that the material of the mechanical part is the ideal material or negative ideal material and then two traditional structural optimization models can be obtained. The sequential unconstrained minimization technique method (SUMT) (Byrne, 2013) is applied to solve the optimization problems and two groups of dimension optimal solutions can be calculated:

$$
\begin{aligned}
& \boldsymbol{X}_{\text {BEST }}:\left\{x_{1}^{\prime}, x_{2}^{\prime}, \ldots x_{n}^{\prime}\right\}, \\
& \boldsymbol{X}_{\text {WORST }}:\left\{x_{1}^{\prime \prime}, x_{2}^{\prime \prime}, \ldots x_{n}^{\prime \prime}\right\} .
\end{aligned}
$$

Step 3: Compare and analyze the two groups of dimension optimal solutions.

1. If $x_{k}{ }^{\prime}=x_{k}{ }^{\prime \prime}=C$ and $C$ is a constant, which proves that the dimension variable $x_{k}$ is not affected by the material variable, then its value can be set as C.

2. If $x_{k}{ }^{\prime} \neq x_{k}{ }^{\prime \prime}$ then the dimension variable $x_{k}$ is affected by the material variable and its value range can be further narrowed to $x_{k}{ }^{\prime} \leq x_{k} \leq x_{k}{ }^{\prime \prime}$.

3. Substitute the values $\boldsymbol{X}_{\mathrm{BEST}}$ and $\boldsymbol{X}_{\mathrm{WORST}}$ into the constraint conditions. If $g_{j}(\boldsymbol{X})=0$ then the optimal solution is just on the constrained boundary $g_{j}(\boldsymbol{X})=0$, and the other constraints have no effect. Otherwise, the optimal solution is the extreme point of the objective function and the other constraints have no effect.

Step 4: Simplify the model and search for the analytical optimal solution for dimension.

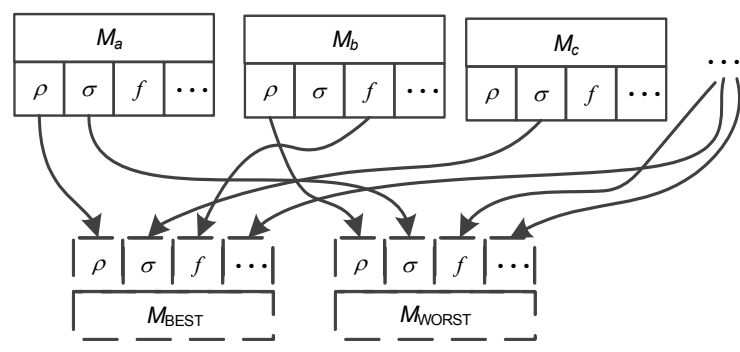

Fig. 3 Construction of the ideal and negative ideal materials 
1. If the optimal solution is the extreme point of the objective function, then the mathematical model can be simplified to:

$$
\left\{\begin{aligned}
& F= \min f_{E_{\mathrm{IP}}}(M) \cdot f_{\rho}(M) \\
& \cdot V\left(C_{1}, C_{2}, \ldots, C_{m}, x_{m+1}, x_{m+2}, \ldots, x_{n}\right), \\
& \boldsymbol{X}= {\left[x_{m+1}, x_{m+2}, \ldots, x_{n}, M\right]^{\mathrm{T}}, } \\
& x_{m+k}^{\prime} \leq x_{m+k} \leq x_{m+k}^{\prime \prime} .
\end{aligned}\right.
$$

Treat the material variable as a constant and compute the partial derivatives of the objective function. Set their values to 0 according to the necessary condition to reach the extreme point:

$$
\nabla F(\boldsymbol{X})=\left[\frac{\partial F}{\partial x_{m+1}} \frac{\partial F}{\partial x_{m+2}} \ldots \frac{\partial F}{\partial x_{n}}\right]^{\mathrm{T}}=0
$$

Then the dimension optimal solutions in analytical form can be calculated:

$$
\left[x_{m+1}, x_{m+2}, \ldots, x_{n}\right]^{\mathrm{T}}=\left[\varphi_{1}(M), \varphi_{2}(M), \ldots, \varphi_{n-m}(M)\right]^{\mathrm{T}} .
$$

2. If the optimal solution is on the constrained boundary $g_{j}(\boldsymbol{X})=0$, then the mathematical model can be simplified to:

$$
\left\{\begin{aligned}
& F= \min f_{E_{\mathrm{IP}}}(M) \cdot f_{\rho}(M) \\
& \cdot V\left(C_{1}, C_{2}, \ldots, C_{m}, x_{m+1}, x_{m+2}, \ldots, x_{n}\right), \\
& \text { s.t. } g_{j}(\boldsymbol{X})=0 \\
& \boldsymbol{X}= {\left[x_{m+1}, x_{m+2}, \ldots, x_{n}, M\right]^{\mathrm{T}} } \\
& x_{m+k}^{\prime} \leq x_{m+k} \leq x_{m+k}^{\prime \prime}
\end{aligned}\right.
$$

This is an optimization problem with equality constraints. Using the Lagrange multiplier method, it can be transformed into an optimization problem without constraints. A new variable $\lambda$, called the Lagrange multiplier, is introduced then the objective function can be transformed into:

$$
\begin{aligned}
F^{\prime}= & \min \left[f_{E_{\mathrm{IP}}}(M) \cdot f_{\rho}(M)\right. \\
& \left.\cdot V\left(C_{1}, C_{2}, \ldots, C_{m}, x_{m+1}, x_{m+2} \ldots, x_{n}\right)+\lambda g_{j}(\boldsymbol{X})\right] .
\end{aligned}
$$

Similar to the method in Eq. (1), the dimension optimal solutions in analytical form can be obtained.
Step 5: Substitute the dimension optimal solutions into the objective function and obtain the result $F=\min C^{\prime} \cdot \xi$, where $C^{\prime}$ is a constant, and $\xi=\Phi\left[f_{\rho}(M)\right.$, $\left.f_{E}(M), \ldots\right]$ is the environmental performance index for the structure, and is a function with the material performance components as its variables. Calculate the environmental performance index $\xi$ of each material in the optional material set and the optimal material with the smallest $\xi$ can be found. Substitute the material into the analytical expression of the dimension optimal solutions and then the dimension optimal values can be calculated. The final result of the optimal material and optimal structure dimensions can be obtained after rounding the dimension values.

\section{Case study}

For mechanical parts which have a short life and need frequent replacement, special attention needs to be paid at the design stage to their environmental impact. In this case study, a design problem of four dowel bars which are symmetrically distributed and vulnerable parts are considered. Fig. 4 presents the structure of the part.

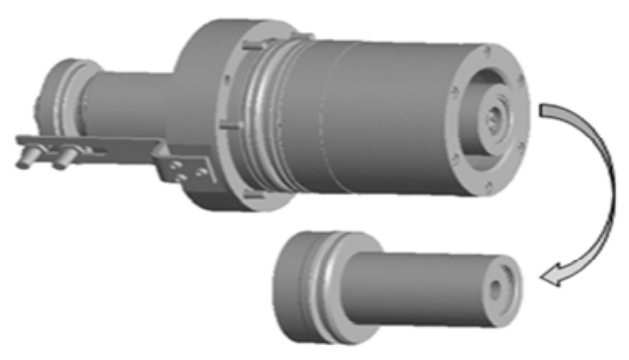

Fig. 4 A dowel bar in motion transfer equipment

Based on engineering analysis, the structure of the part is simplified focusing on the key dimensions. Fig. 5 presents a diagrammatic sketch of the key dimensions, constraints, and load for the part. According to the working condition, the peak value of the torque $M_{1}=16 \mathrm{~N} \cdot \mathrm{m}$, the maximum stress on the left end $F=20000 \mathrm{~N}$, and $M_{2}=2 F \cdot L_{1}$. The allowable flexibility $[y]=0.05 \mathrm{~mm}$, the allowable torsional stiffness $[\varphi]=0.083^{\circ} / \mathrm{m}$, and the allowable steering angle $[\theta]=0.0025^{\circ}$. The value ranges of the dimensions are: $80 \mathrm{~mm} \leq L_{1} \leq 160 \mathrm{~mm}, 350 \mathrm{~mm} \leq L_{2} \leq 600 \mathrm{~mm}, 80 \mathrm{~mm}$ $\leq D_{1} \leq 160 \mathrm{~mm}$, and $70 \mathrm{~mm} \leq D_{2} \leq 150 \mathrm{~mm}$. 


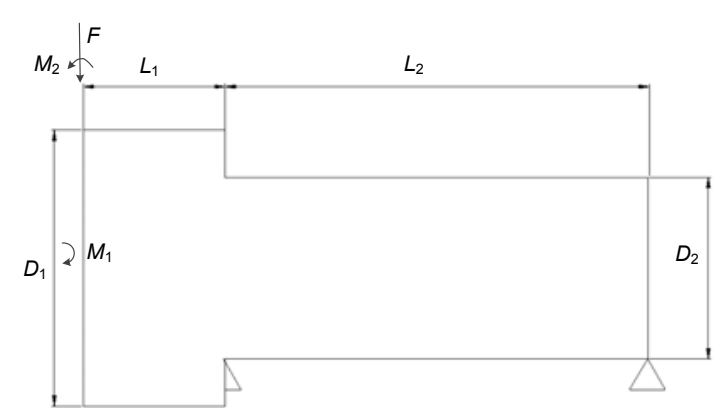

Fig. 5 Diagrammatic sketch of the key dimensions, constraints, and load stress

The density $\rho$, elastic modulus $E$, and shear modulus $G$ are the related material properties for this part.

Using the method proposed in this study, a design process integrating structural optimization and material selection is carried out.

First, construct the hybrid variable including the material variable:

$$
\boldsymbol{X}=\left[\begin{array}{c}
D_{1} \\
D_{2} \\
L_{1} \\
L_{2} \\
M
\end{array}\right]=\left[\begin{array}{lllll}
x_{1} & x_{2} & x_{3} & x_{4} & M
\end{array}\right]^{\mathrm{T}},
$$

where $M=\left\{E_{\mathrm{IP}}, \rho, E, G\right\}$.

Establish the objective function with the aim of minimizing the environmental impact:

$$
F(x)=\min \frac{\pi \rho E_{\mathrm{IP}}}{4}\left(x_{1}^{2} x_{3}+x_{2}^{2} x_{4}\right)
$$

Determine the constraints according to the design requirements:

1. Stiffness constraints

Calculate the maximum displacement of the left end under the action of $F$ and $M_{2}$ using superposition method. Considering the allowable flexibility, the stiffness constraints can be obtained:

$$
g_{1}(x)=\frac{64 F x_{3}^{2}}{3 \pi E}\left(\frac{4 x_{3}}{x_{1}^{4}}+\frac{3 x_{4}}{x_{2}^{4}}\right)-[y] \leq 0
$$

2. Torsional deformation constraints
Calculate the torsion angle under the action of $M_{1}$ and the torsional deformation constraints can be obtained:

$$
g_{2}(x)=\frac{180}{\pi} \cdot \frac{M_{1}}{G \pi x_{2}^{4} / 32}-[\varphi] \leq 0 .
$$

3. Steering angle constraints

The maximum steering angle is on the right end of the cantilever, so the steering angle constraints can be obtained:

$$
g_{3}(x)=\frac{F x_{3} x_{4}}{3 E x_{2}^{4}}-[\theta] \leq 0
$$

4. Dimension constraints

The dimension constraints are derived from the design range of each structure dimension: $g_{4}(x)$ $=80-x_{1} \leq 0, \quad g_{5}(x)=x_{1}-160 \leq 0, \quad g_{6}(x)=70-x_{2} \leq 0, \quad g_{7}(x)$ $=x_{2}-150 \leq 0, \quad g_{8}(x)=80-x_{3} \leq 0, \quad g_{9}(x)=x_{3}-160 \leq 0, \quad g_{10}(x)$ $=350-x_{4} \leq 0$, and $g_{11}(x)=x_{4}-600 \leq 0$.

According to the actual requirements, this dowel bar should have high strength, good abrasion resistance, long service life, easy cutting, and be easy to recycle.

Therefore, the qualitative screening conditions can be obtained:

$M \in\{$ Metal materials $\} \& \& M \in\{$ Recyclable material $\}$.

Transform the constraints into:

$$
\left\{\begin{array}{l}
E \geq \frac{64 F x_{3}^{2}}{3 \pi[y]}\left(\frac{4 x_{3}}{x_{1}^{4}}+\frac{3 x_{4}}{x_{2}^{4}}\right), \\
E \geq \frac{F x_{3} x_{4}}{3[\theta] x_{2}^{4}}, \\
G \geq \frac{180}{\pi} \cdot \frac{M_{0}}{\pi[\varphi] x_{2}^{4} / 32} .
\end{array}\right.
$$

Calculate the extreme values of the polynomial on the right side of inequality Eq. (18), and the quantitative screening conditions can be obtained:

$$
E \geq 12.2 \mathrm{GPa} \& \& G \geq 2.2 \mathrm{GPa} .
$$

Based on the screening conditions Eqs. (17) and (19) and choosing some commonly used materials 
according to practical experience, the optional materials set is obtained. Using SimaPro7.3 to analyze the environmental impact of each material, we can obtain the properties table (Table 1) for the optional materials.

For best results, the material should have smaller $\rho$ and $E_{\mathrm{IP}}$ with greater $E$ and $G$. Accordingly, we can build the ideal material and the negative material with the boundary values of these properties in the optional materials set:

$$
\begin{aligned}
& M_{\mathrm{BEST}}=\left\{\rho, E, G, E_{\mathrm{IP}}\right\}=\{2700,210,80,86\}, \\
& M_{\mathrm{WORST}}=\left\{\rho, E, G, E_{\mathrm{IP}}\right\}=\{8900,70,26,1400\} .
\end{aligned}
$$

Table 1 Properties index of the optional materials

\begin{tabular}{lcccc}
\hline \multicolumn{1}{c}{ Material } & $\rho\left(\mathrm{kg} / \mathrm{m}^{3}\right)$ & $E(\mathrm{GPa})$ & $G(\mathrm{GPa})$ & $E_{\mathrm{IP}}(\mathrm{mPt} / \mathrm{kg})$ \\
\hline 45 Steel & 7900 & 210 & 80 & 86 \\
Brass & 8900 & 93 & 35 & 1500 \\
$\begin{array}{l}\text { Aluminum } \\
\text { alloy }\end{array}$ & 2700 & 70 & 26 & 800 \\
$\begin{array}{c}\text { Ductile iron } \\
\text { Manganese }\end{array}$ & 7200 & 150 & 74 & 260 \\
$\quad$ bronze & 8600 & 108 & 39 & 1910 \\
Zinc alloy & 7300 & 82 & 31 & 1320 \\
\hline
\end{tabular}

Plug Eq. (20) into the optimization model and calculate two groups of dimension solutions:

$$
\begin{aligned}
& \boldsymbol{X}_{\text {BEST }}=[107.1574,102.1407,80,350]^{\mathrm{T}}, \\
& \boldsymbol{X}_{\text {WORST }}=[141.0271,134.4248,80,350]^{\mathrm{T}} .
\end{aligned}
$$

From the comparative analysis, we know that $x_{3}$ and $x_{4}$ are not affected by the material variable so their values can be set as constants while $x_{1}$ and $x_{2}$ are affected by the material variable. Substituting the values $\boldsymbol{X}_{\mathrm{BEST}}$ and $\boldsymbol{X}_{\mathrm{WORST}}$ into the constraint conditions, we obtain $g_{j}(x)=0$, which means that the optimal solution is just on this constrained boundary, so we can simplify the model as

$$
\left\{\begin{array}{l}
F(x)=\min \frac{\pi \rho E_{\mathrm{IP}}}{4}\left(x_{1}^{2} x_{3}+x_{2}^{2} x_{4}\right), \\
\frac{64 F x_{3}^{2}}{3 \pi E}\left(\frac{4 x_{3}}{x_{1}^{4}}+\frac{3 x_{4}}{x_{2}^{4}}\right)-[y]=0 .
\end{array}\right.
$$

Introducing the Lagrange multiplier $\lambda$, the problem is transformed into an optimization problem without constraints:

$$
\begin{aligned}
& F^{\prime}(x, \lambda)=\frac{\pi \rho E_{\mathrm{IP}}}{4}\left(x_{1}^{2} x_{3}+x_{2}^{2} x_{4}\right) \\
& +\lambda\left[\frac{64 F x_{3}^{4}}{3 \pi E}\left(\frac{4 x_{3}}{x_{1}^{4}}+\frac{3 x_{4}}{x_{2}^{4}}\right)-[y]\right] .
\end{aligned}
$$

With the necessary condition to reach the extreme point we can obtain:

$$
\left\{\begin{array}{l}
\frac{\partial F^{\prime}(x, \lambda)}{x_{1}}=0, \\
\frac{\partial F^{\prime}(x, \lambda)}{x_{2}}=0, \\
\frac{\partial F^{\prime}(x, \lambda)}{\lambda}=0 .
\end{array}\right.
$$

Calculate out:

$$
x_{1}=6.33 \sqrt[4]{\frac{64 F x_{3}^{2}}{3 \pi E[y]}}, \quad x_{2}=6.02 \sqrt[4]{\frac{64 F x_{3}^{2}}{3 \pi E[y]}},
$$

and plug them into the objective function:

$$
\begin{aligned}
F(x)= & \min \left\{\frac { \pi \rho E _ { \mathrm { IP } } } { 4 } \left(6.33^{2} x_{3} \sqrt{\frac{64 F x_{3}^{2}}{3 \pi E[y]}}\right.\right. \\
& \left.\left.+6.02^{2} x_{4} \sqrt{\frac{64 F x_{3}^{2}}{3 \pi E[y]}}\right)\right\} \\
= & \min \left\{\frac{\pi}{4}\left(6.33^{2} x_{3}+6.02^{2} x_{4}\right) \sqrt{\frac{64 F x_{3}^{2}}{3 \pi[y]}} \cdot \frac{\rho E_{\mathrm{IP}}}{\sqrt{E}}\right\},
\end{aligned}
$$

which can be expressed as $F=\min C^{\prime} \cdot \xi$, where $C^{\prime}=\frac{\pi}{4}\left(6.33^{2} x_{3}+6.02^{2} x_{4}\right) \sqrt{\frac{64 F x_{3}^{2}}{3 \pi[y]}}$ is a constant, and $\xi=\frac{\rho E_{\mathrm{IP}}}{\sqrt{E}}$ is the environmental performance index for this part.

Calculate and compare each $\xi$ of the optional materials. Table 2 lists the values of $\xi$ for the materials chosen previously.

Through the comparison, 45 Steel, which has the smallest $\xi$, is the best choice. The dimension solution can be calculated by substituting the related performance values of 45 Steel into Eq. (25): 


$$
\begin{aligned}
& x_{1}=6.33 \sqrt[4]{\frac{64 F x_{3}^{2}}{3 \pi E[y]}}=107.1574, \\
& x_{2}=6.02 \sqrt[4]{\frac{64 F x_{3}^{2}}{3 \pi E[y]}}=102.1407 .
\end{aligned}
$$

We can obtain the whole dimension values: $X=[107.1574,102.1407,80,350]^{\mathrm{T}}$. After rounding the dimension values, the final design proposal of this part can be determined: 45 Steel is chosen as the material with the dimension $D_{1}=108 \mathrm{~mm}, D_{2}=$ $103 \mathrm{~mm}, L_{1}=80 \mathrm{~mm}$, and $L_{2}=350 \mathrm{~mm}$.

Table 2 Environmental performance indexes of the optional materials

\begin{tabular}{lr}
\hline \multicolumn{1}{c}{ Material } & $\xi=\frac{\rho E_{\mathrm{IP}}}{\sqrt{E}}$ \\
\hline 45 Steel & 46883.1 \\
Brass & 1384330.5 \\
Aluminum alloy & 258169.4 \\
Ductile iron & 152848.2 \\
Manganese bronze & 1580592.6 \\
Zinc alloy & 1064118.2 \\
\hline
\end{tabular}

\section{Summary and conclusions}

To provide a greener approach to mechanical part design, a method for combining structural optimization and material selection is proposed. The distinguishing features of this paper are:

1. A hybrid optimization model was built, introducing a discrete variable of material into the ordinary structure optimization model. This new optimization model can provide a more reasonable proposal by integrating structural optimization with environmental impact assessment.

2. An optional material set was obtained by combining measures of qualitative and quantitative screening, while the lifecycle environmental impacts of the materials were quantified using the method of Eco-indicator 99.

3. The hybrid model was simplified by calculating and comparing the optimal structure solutions with selected ideal and negative ideal materials. The material environmental performance index was separated using analytic method. Through comparison of the material environmental performance index for every material in the optional material set, the optimal material can be found and the structure solutions calculated. This method was shown to be practical with the case study of a dowel bar design process. The final results showed that $L_{1}$ and $L_{2}$ remained the same with the optimal solutions of the models, which were built by selecting the ideal and negative ideal materials, while $D_{1}$ and $D_{2}$ were within the dimension ranges. The environmental impact of each material has a significant effect on the optimal structural solution. Therefore, it is important to study them together so that they can be matched more reasonably for green design.

This paper focuses on the environmental impact of mechanical parts at the design stage. It represents a new approach to green design with structural optimization and material selection integrated together. But the method has limitations: it is suitable only for structural dimension optimization with a single objective and an easy mathematical model. More work needs to be done to develop a generic method and multi-objective optimization should be considered. In future work, we will include the material variable in complex structural optimization by finite element analysis method.

\section{References}

Almeida, C.M.V.B., Rodrigues, A.J.M., Bonilla, S.H., Giannetti, B.F., 2010. Emergy as a tool for Ecodesign: evaluating materials selection for beverage packages in Brazil. Journal of Cleaner Production, 18(1):32-43. [doi:10. 1016/j.jclepro.2009.03.019]

Amen, R., Vomacka, P., 2001. Case-based reasoning as a tool for materials selection. Materials \& Design, 22(5):353358. [doi:10.1016/S0261-3069(00)00105-9]

Ashby, M.F., 2000. Multi-objective optimization in material design and selection. Acta Materialia, 48(1):359-369. [doi:10.1016/S1359-6454(99)00304-3]

Ashby, M.F., Johnson, K.W., 2002. Material and Design: the Art and Science of Material Selection in Product Design. Butterworth-Heinemann, Oxford, UK.

Byrne, C.L., 2013. Alternating minimization as sequential unconstrained minimization: A survey. Journal of Optimization Theory and Applications, 156(3):554-566. [doi:10.1007/s10957-012-0134-2]

Chu, C.H., Luh, Y.P., Li, T.C., Chen, H., 2009. Economical green product design based on simplified computer-aided product structure variation. Computers in Industry, 60(7): 485-500. [doi:10.1016/j.compind.2009.02.003]

Deng, Y.M., Edwards, K.L., 2007. The role of materials 
identification and selection in engineering design. Materials \& Design, 28(1):131-139. [doi:10.1016/j.matdes. 2005.05.003]

Hambali, A., Sapuan, S.M., Ismail, N., Nukman, Y., 2010. Materials selection of polymeric composite automotive bumper beam using analytical hierarchy process. Journal of Central South University of Technology, 17(2): 244-256. [doi:10.1007/s11771-010-0038-y]

Holloway, L., 1998. Materials selection for optimal environmental impact in mechanical design. Materials \& Design, 19(4):133-143. [doi:10.1016/S0261-3069(98)00031-4]

Huang, H.H., Liu, Z.F., Zhang, L., Sutherland, J.W., 2009. Materials selection for environmentally conscious design via a proposed life cycle environmental performance index. The International Journal of Advanced Manufacturing Technology, 44(11-12):1073-1082. [doi:10.1007/ s00170-009-1935-9]

Jahan, A., Ismail, M.Y., Mustapha, F., Sapuan, S.M., 2010. Material selection based on ordinal data. Materials \& Design, 31(7):3180-3187. [doi:10.1016/j.matdes.2010.02. 024]

Jahan, A., Mustapha, F., Ismail, M.Y., Sapuan, S.M., Bahraminasab, M., 2011. A comprehensive VIKOR method for material selection. Materials \& Design, 32(3):12151221. [doi:10.1016/j.matdes.2010.10.015]

Khabbaz, R.S., Manshadi, B.D., Abedian, A., Mahmudi, R., 2009. A simplified fuzzy logic approach for materials selection in mechanical engineering design. Materials \& Design, 30(3):687-697. [doi:10.1016/j.matdes.2008.05. 026]

Kurk, F., Eagan, P., 2008. The value of adding design-forthe-environment to pollution prevention assistance options. Journal of Cleaner Production, 16(6):722-726. [doi:10.1016/j.jclepro.2007.02.022]

Natalia, S.E., Maria, B.G.C., Prabhu, V.K., 2004. Material selection for an automotive structure by integrating structural optimization with environmental impact assessment. Materials \& Design, 25(8):689-698. [doi:10. 1016/j.matdes.2004.02.021]

Natalia, S.E., Kirill, G.K., Jan, L.S., 2002. Materials selection combined with optimal structural design: concept and some results. Materials \& Design, 23(5):459-470. [doi:10.1016/S0261-3069(02)00019-5]

Nagy, A.P., Abdalla, M.M., Gurdal, Z., 2010. Isogeometric sizing and shape optimisation of beam structures. Computer Methods in Applied Mechanics and Engineering, 199(17-20):1216-1230. [doi:10.1016/j.cma.2009.12.010]

Peter, W.C., Anders, K., 2009. An Introduction to Structural Optimization. Springer Verlag, Berlin Heidelberg.

Rao, R.V., 2007. Decision Making in the Manufacturing
Environment Using Graph Theory and Fuzzy Multiple Attribute Decision Making Methods. Springer Verlag, London.

Rao, R.V., Davim, J.P., 2008. A decision-making framework model for material selection using a combined multiple attribute decision-making method. The International Journal of Advanced Manufacturing Technology, 35(7-8):751-760. [doi:10.1007/s00170-006-0752-7]

Rao, R.V., Patel, B.K., 2010. A subjective and objective integrated multiple attribute decision making method for material selection. Materials \& Design, 31(10):4738-4747. [doi:10.1016/j.matdes.2010.05.014]

Ribeiro, I., Pecas, P., Silva, A., Henriques, E., 2008. Life cycle engineering methodology applied to material selection, a fender case study. Journal of Cleaner Production, 16(17): 1887-1899. [doi:10.1016/j.jclepro.2008.01.002]

Rydh, C.J., Sun, M., 2005. Life cycle inventory data for materials grouped according to environmental and material properties. Journal of Cleaner Production, 13(13-14): 1258-1268. [doi:10.1016/j.jclepro.2005.05.012]

Sapuan, S.M., 2001. A knowledge-based system for materials selection in mechanical engineering design. Materials \& Design, 22(8):687-695. [doi:10.1016/S0261-3069(00)001 08-4]

Sapuan, S.M., Abdalla, H.S., 1998. A prototype knowledgebased system for the material selection of polymericbased composites for automotive components. Composites Part A: Applied Science and Manufacturing, 29(7): 731-742. [doi:10.1016/S1359-835X(98)00049-9]

Song, X., Baldwin, J.D., 1999. A novel node-based structural shape optimization algorithm. Computers \& Structures, 70(5):569-581. [doi:10.1016/S0045-7949(98)00210-7]

Sun, M., Rydh, C.J., Kaebernick, H., 2003. Material grouping for simplified product life cycle assessment. The Journal of Sustainable Product Design, 3(1-2):45-58. [doi:10. 1023/B:JSPD.0000035558.27697.02]

Wall, W.A., Frenzel, M.A., Cyron, C., 2008. Isogeometric structural shape optimization. Computer Methods in Applied Mechanics and Engineering, 197(33-40):2976-2988. [doi:10.1016/j.cma.2008.01.025]

Weaver, P.M., Ashby, M.F., Burgess, S., Shibaike, N., 1996. Selection of materials to reduce environmental impact: a case study on refrigerator insulation. Materials \& Design, 17(1):11-17. [doi:10.1016/0261-3069(96)00024-6]

Zha, X.F., 2005. A web-based advisory system for process and material selection in concurrent product design for a manufacturing environment. The International Journal of Advanced Manufacturing Technology, 25(3-4):233-243. [doi:10.1007/s00170-003-1838-0] 